\title{
El acceso a la información como eje principal de la responsabilidad social del profesional de la información
}

\author{
Ada Suyin Sosa Solano \\ Célia Celia Revilandia Costa Seabra \\ Elmira Luzia Melo Soares Simeão \\ Universidade de Brasília - UnB, Brasil
}

REVIEW

\begin{abstract}
Resumen
Objetivo. Este artículo reflexiona sobre la relación entre la responsabilidad social de las Ciencias de la Información (Cl) y sus profesionales en la actualidad, desde un enfoque de derechos humanos e inclusión en la sociedad del conocimiento.

Método. Se realizó una revisión sistemática de la literatura de los principales autores que tratan la ciencia de la información con un enfoque social.

Resultados. Se identificaron diversos argumentos que confirman el papel desempeñado por los profesionales de la información como promotores y vigilantes del Derecho de Acceso a la Información.

Conclusiones. Se concluye que el acceso a la información es el eje principal de la responsabilidad social de los profesionales de la información.
\end{abstract}

Palabras-clave

Acceso a la información ; Responsabilidad social ; Profesional de la información ; Ciencia de la Información ; Inclusión social ; Sociedad del conocimiento

\section{Access to information as the main axis of the social responsibility of information professionals}

\begin{abstract}
Objective. This paper reflects about relationship between social responsibility of Information Science (IS) and its professionals at present, from a focus on human rights and inclusion in the knowledge society. .

Method. Literature review of the main authors that treat information science with a social focus.

Results. They were identified several arguments that confirm the role played by the information professionals as promoters and guards the Right of Access to Information.

Conclusions. We believe that providing access to information is the main axis of the social responsibility of information professionals.
\end{abstract}

Keywords

Access to information; Social responsibility ; Professional information ; Information Science ; Social inclusion ; Knowledge society

\section{Introducción}

El desarrollo actual de nuestra sociedad, basada en una economía que reconoce el amplio valor de la información como elemento transformador de la actividad económica y social, invita a los profesionales que participamos de la Ciencia de la Información $(\mathrm{Cl})$ a reflexionar sobre las implicancias de nuestro quehacer en este nuevo escenario global.

De acuerdo Castells (2000, p. 548-559) nos encontramos en medio de una transformación histórica, que se viene gestando desde las últimas décadas, la cual está generando un modelo de sociedad en la cual la información es el motor del cambio social. Un paradigma que él denomina la sociedad red y que otros denominan sociedad de la información, o sociedad del conocimiento. 
Sin embargo este nuevo mundo, caracterizado por la acumulación de información y deslumbrado con las tecnologías de la información y comunicación, no es perfecto. Cada día es más evidente la brecha entre los que tienen acceso a la información y el conocimiento tecnológico y los que se encuentran al margen de las nuevas tecnologías de información y comunicación (TIC).

Distintas disciplinas de las Ciencias Sociales, incluida la $\mathrm{Cl}$ se encuentran convocadas a plantear sus propuestas en la búsqueda de una sociedad más equitativa para todos independientemente de sus diferencias económicas, culturales y físicas. No sólo la exclusión social, sino también económica, y aún más, la exclusión a personas con discapacidad, son temas que deben ser trabajados desde la $\mathrm{Cl}$.

El propósito de este ensayo es presentar desde la perspectiva de la responsabilidad social, los aportes de la $\mathrm{Cl}$ y sus profesionales frente a los nuevos retos de la Sociedad del Conocimiento, para lo cual se desarrollará con mayor profundidad el tema de la inclusión, partiendo de los conceptos más amplios para profundizar en la inclusión de las personas con deficiencia.

\section{Derechos humanos, Ciencia de la Información e inclusión social}

La Cl, "como muchas otras áreas interdisciplinares, tuvo sus orígenes en el centro de la revolución científica y técnica que vino luego de la Segunda Guerra Mundial” (SARACEVIC, 1996, p.42); es en ese sentido contemporánea al contexto de planteamiento de los Derechos Humanos desde una perspectiva mundial. Así con el auge de la reorganización social y en el contexto de la post guerra, la contribución de la $\mathrm{Cl}$ estuvo dirigida al desenvolvimiento de estrategias científicas para garantizar el acceso a información, esto como parte de un conjunto de derechos defendidos y promovidos socialmente.

En este sentido, prosigue Saracevic, la $\mathrm{Cl}$ tiene tres grandes características generales: su naturaleza interdisciplinar; su relación directa con las TIC; y su participación activa y deliberada en la evolución de la sociedad de la información. Este último punto corresponde a la dimensión social de la $\mathrm{Cl}$, y puede vincularse directamente a la Declaración Universal de Derechos Humanos - DUDH (1948), que en su Artículo 19 expresa que:

Todo individuo tiene derecho a la libertad de opinión y de expresión; este derecho incluye el de no ser molestado a causa de sus opiniones, el de investigar y recibir informaciones y opiniones, y el de difundirlas, sin limitación de fronteras, por cualquier medio de expresión (ORGANIZAÇÃO DAS NAÇÕES UNIDAS, 1948).

Tempo después otros organismos internacionales, como la Federación Internacional de Asociaciones de Bibliotecarios e Instituciones - IFLA, apoyándose en la DUDH han declarado que:

[...] Los seres humanos tienen el derecho fundamental de tener acceso a las expresiones del conocimiento, del pensamiento creativo y de la actividad intelectual, y de expresar públicamente sus opiniones [...] El derecho al conocimiento y a la libertad de expresión son dos aspectos del mismo principio. El derecho al conocimiento es un requisito para la libertad de pensamiento y de conciencia; la libertad de acceso a la información es una condición imprescindible para el ejercicio de la libertad de pensamiento y de la libertad de expresión (IFLA, 1999).

De esta forma se ha venido sustentando lo que actualmente conocemos como Derecho al Acceso a la Información, enmarcado como un derecho fundamental, el cual lamentablemente no se da de igual forma para todas las personas. Afortunadamente la evolución social, científica y técnica, como plantea Cuevas (2011), ha generado nuevos producen nuevos derechos complementarios que refuerzan su propósito:

[...] 1.- Comunicación y a la información: información completa y verdadera; derecho al acceso a información de relevancia para la humanidad, derecho de acceso a los medios técnicos de comunicación públicos y privados... etc. 2.-Derechos en la red: derechos informáticos, derechos a la vida privada en la red, a la propiedad intelectual e industrial en la red. 3.Derechos de los menores (CUEVAS, 2011, p.30).

Por su parte este conjunto de derechos propicia el enfoque de desarrollo humano y por ende el de inclusión social de y para todas las personas. En esa misma lectura, la pobreza, la marginalización, la falta de acceso a la información y a las tecnologías de información, el analfabetismo y la falta de acceso a los servicios de salud y educación son considerados formas de exclusión que atentan contra el desarrollo de la sociedad. Es por ello que 
desde el Programa de las Naciones Unidas para el Desarrollo - PNUD se viene promoviendo actualmente los Objetivos de Desarrollo del Milenio - ODM (2000) para mejorar estas formas de exclusión.

\section{Enfoque social de la Ciencia de la Información}

Diversos investigadores (BELKIN, 1878; WERSIG \& NEVELING, 1975; CAPURRO \& HJORLAND, 2007) coinciden en que la ciencia inherentemente responde a un propósito social, toda vez que sus esfuerzos están enfocados a alcanzar el bienestar social. Para Bunge(1973, p. 6) "la ciencia como actividad —como investigación - pertenece a la vida social; en cuanto se la aplica al mejoramiento de nuestro medio natural y artificial, a la invención y manufactura de bienes materiales y culturales, la ciencia se convierte en tecnología".

En ese mismo sentido Tomanik (2004, p. 169) precisa que la actividad científica, como cualquier actividad humana, es realizada dentro de un contexto social, y es influenciada, o incluso determinada por este contexto. Cada vez que la $\mathrm{Cl}$ es postulada como una ciencia es inmediatamente vinculada al conjunto de las Ciencias Sociales, y esto conlleva a la vez a plantear el propósito social de la misma. Así Almeida (2007 p. 72) añade que la $\mathrm{Cl}$ es un campo social de producción del conocimiento llevado a las cuestiones vinculadas a la información.

Estas ideas se complementa con lo expresado por Renault (2007, p. 144) quien nos dice que lo que nos debe interesar es que la $\mathrm{Cl}$ mantenga la perspectiva de comprensión de la manera en que el ser humano utiliza el conocimiento, al igual que la idea de la $\mathrm{Cl}$ como una ciencia del orden de las cuestiones sociales.

Tenemos entonces que la $\mathrm{Cl}$, como área de investigación, se coloca como eje disciplinar que considera tanto los aspectos de la tecnología y del registro del conocimiento, asociadas a diversos campos, como a las reglas clásicas de organización y tratamiento de la información, de donde se tiene el origen del desarrollo del área.

Bajo la perspectiva de esta responsabilidad, adoptando el enfoque de Thompson (1998), también recaen los cuidados de los contornos semánticos, metodológicos y de la acción investigadora, entendiendo que la información es un "objeto", con diversos contornos y, por lo tanto, permite distintas interpretaciones, unas diferentes, otras similares. En el campo de la comunicación, por ejemplo, el tratamiento técnico del mensaje y de la comunicación de la información es un ejercicio permanente:

Desde las más antigua formas de comunicación gestual y de uso del lenguaje hasta los progresos más recientes de la tecnología de computación, de producción, de almacenamiento y de circulación de la información y de contenido simbólicos han sido aspectos centrales de la vida social ". (THOMPSON, 1998, p. 19)

Planteando un análisis más amplio de la Cl en el contexto social, Le Coadic (1996), propone la definición de los límites de esta ciencia de forma pragmática, hasta alcanzar la comprensión de ella en la óptica de los especialistas que vienen de otros campos:

[...] tiene como objetivo el estudio de las características generales de la información (naturaleza, origen y efectos), es decir, más precisamente, el análisis de los procesos de construcción, comunicación y uso de la información (LE COADIC, 1996, p.26).

Se encuentran así en la $\mathrm{Cl}$, elementos de un paradigma objetivo, donde la creación de los productos y sistemas para las estrategias de impacto social, son previstas y estudiadas objetivamente al igual que las metodologías e intervenciones. Se evidencia de esta forma la relación inmediata de la $\mathrm{Cl}$ con la sociedad desde sus inicios.

\section{Responsabilidad social de la Ciencia de la Información}

Empezaremos citando a Nietzsche (1887) que describe la responsabilidad como el hecho de responder por sus actos, frente a los demás, y responder por el futuro en general. Esta capacidad es la de un ser que tiene la facultad de hacer promesas y cumplirlas, es decir que puede "disponer por anticipación del futuro".

Por su parte, Freire (1983), afirma que la primera condición para que una persona pueda asumir un acto comprometido (responsable) debe ser capaz de actuar y reflexionar.

Solamente un ser que es capaz de emerger de su contexto, de "alejarse" de él para quedar con él; capaz de admirarlo para, objetivándolo, transformarlo, y transformándolo, saberse transformado por su propia creación; un ser que es y está siendo en el tiempo que es suyo, un ser histórico... solamente éste es capaz, por todo esto, de comprometerse. (FREIRE, 1983. p.3) 
Con el pasar de los años el término responsabilidad fue ganando mayor cobertura hasta llegar a hablar del término de responsabilidad social, pudiendo utilizar el término tanto para las empresas como para las instituciones sociales y gubernamentales. Para nuestro caso abordaremos la relación ética de los profesionales quienes participamos de la $\mathrm{Cl}$, desde el enfoque de la Responsabilidad Social (RS). Esta fue definida de forma pionera por la Norma Técnica Brasilera: NBR 16000 (norma de responsabilidade social) como:

\begin{abstract}
[...] Esta responsabilidad puede ser "negativa", significando que hay responsabilidad de abstenerse de actuar (actitud de "abstención") o puede ser "positiva", significando que hay una responsabilidad de actuar. (actitud proactiva) (ASSOCIAÇÃO BRASILEIRA DE NORMAS TÉCNICAS, 2004).
\end{abstract}

De otra parte tenemos que la Organización Internacional para la Estandarización (ISO por sus siglas en inglés) plantea la ISO 26000 como guía que establece las líneas a seguir en materia de RS; y la define como:

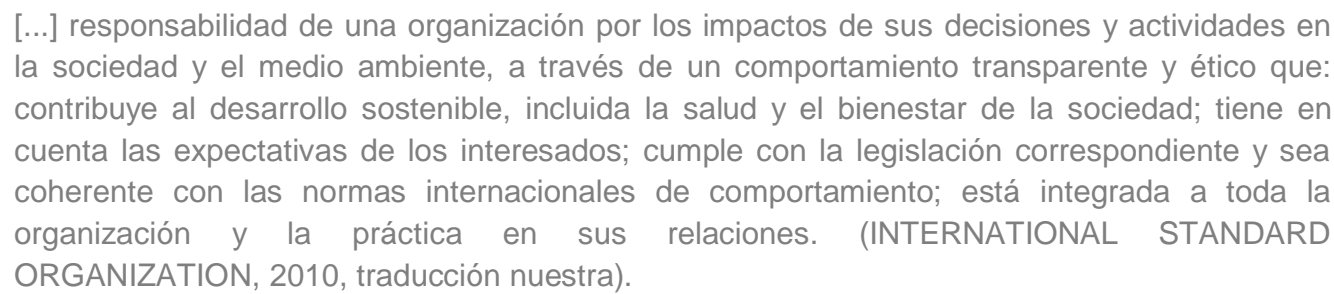

En estas definiciones el criterio de RS se plantea desde la actitud de las personas, o un grupo social de cara a mantener un equilibrio con su entorno.

A partir de estos conceptos podemos postular que la responsabilidad social de la $\mathrm{Cl}$ se refiere al comportamiento ético que la comunidad profesional de esta área tiene con el fin de mantener un equilibrio entre la actividad económica y el ecosistema de nuestra sociedad.

Múnera (2011, p. 138) respalda esta perspectiva, manifestando que:

[...] resulta interesante adoptar a la responsabilidad social del profesional de la Ciencia de la Información, como el impacto positivo que genera su desempeño en la sociedad, mediante la realización de dinámicas que contribuyan a elevar la calidad de vida de las comunidades donde se proyecte, así como el fomentar principios y valores que conduzcan al crecimiento y desarrollo de los seres humanos, como protagonistas de la construcción de una mejor sociedad (MÚNERA TORRES, 2011, p. 138).

Además Múnera (2011, p. 144) alega que la RS se constituye en un componente que es parte del desempeño de los profesionales de la $\mathrm{Cl}$, en los diferentes campos de acción en los que se proyecten. El ofrecer la posibilidad de generar nuevas opciones de desarrollo cultural para las personas desde espacios como una biblioteca, un archivo, un museo o un centro de documentación, contribuye de manera significativa a elevar la calidad de vida de las comunidades y favorece a la construcción de una mejor sociedad.

\title{
4 El acceso a la información como responsabilidad social
}

Hace más de tres décadas Wersig y Neveling (1975, p. 11) habían identificado que la transferencia de conocimiento generaba una circunstancia de "responsabilidad social" y en consecuencia este era el motivo de ser de la $\mathrm{Cl}$, el resolver los problemas de transferencia de conocimiento, resolver los problemas de acceso al conocimiento, expresado de una forma amplia: resolver los problemas de acceso a la información.

Esto es respaldado por Saracevic (1996, p. 60) quien manifiesta que la tarea masiva de la Cl es hacer más accesible el acervo creciente de conocimiento.

Tenemos que tanto los planteamientos de Wersig, Neveling y Saracevic coinciden en que la responsabilidad social de la $\mathrm{Cl}$ se centra en resolver los problemas de acceso a la información. De esta manera, y enfocado como un derecho, el acceso a la información puede ser usado como un instrumento para garantizar el cumplimiento de otros derechos esenciales del ser humano.

La dimensión de esta responsabilidad social es amplia y va más allá de los temas que suele abordar la $\mathrm{Cl}$ de forma frecuente. Como ejemplo, tan solo la defensa del derecho al acceso a la información puede inclusive vincularse a enfoques sociales amplios como el de la democratización de la información. Al respecto Múnera (2011, p. 145) nos recuerda que los servicios de información pública desempeñan desde siempre un rol 
importante al constituir nodos que canalizan los derechos de acceso a la información, al conocimiento y a la cultura, y por ello son necesarios en la construcción de la democracia.

Además, gran parte de la responsabilidad social y de la inclusión social implica la necesidad de asumir un papel dinámico y movilizador del acceso a la información para las personas con deficiencias y limitaciones. Esto significa entonces que todo profesional de la información deberá preocuparse en fomentar el acceso físico y digital de la información y crear los vínculos necesarios para que todas las personas sin ninguna excepción puedan tener acceso a la diversidad de información

Así mismo, actividades paralelas o a fines de este rol, como la alfabetización informacional, la alfabetización digital, así como el desarrollo de capacidades para el manejo de información, constituyen actividades de inclusión social que buscan enfrentar la exclusión que se genera con fenómenos como la brecha digital.

Freire (2004b, p. 192) destaca este aspecto manifestando que la democratización del acceso a las tecnologías digitales de información y comunicación debería ser vista como elemento fundamental en las políticas de inclusión social.

Estos argumentos evidencian que el acceso a la información es el eje principal de la responsabilidad social de la $\mathrm{Cl}$ y sus profesionales. Trasladaremos a continuación el significado del acceso a la información a nuestro entorno actual, a la sociedad de la información y a las sociedades del conocimiento.

\title{
5 Acceso a la información en la Sociedad del Conocimiento
}

La UNESCO en su Informe Mundial (2005, p. 17) nos brinda las siguientes precisiones sobre la Sociedad del Conocimiento:

\begin{abstract}
La noción de sociedad de la información se basa en los progresos tecnológicos. En cambio, el concepto de sociedades del conocimiento comprende dimensiones sociales, éticas y políticas mucho más vastas. El hecho de que nos refiramos a sociedades, en plural, no se debe al azar, sino a la intención de rechazar la unicidad de un modelo "listo para su uso" que no tenga suficientemente en cuenta la diversidad cultural y lingüística, único elemento que nos permite a todos reconocernos en los cambios que se están produciendo actualmente. Hay siempre diferentes formas de conocimiento y cultura que intervienen en la edificación de las sociedades, comprendidas aquellas muy influidas por el progreso científico y técnico moderno (UNESCO, 2005, p. 17).
\end{abstract}

Si bien la sociedad de la información está construida sobre la estructura de la tecnología de la información, toma sentido y cumple su función, sobre el principio del acceso a la información. Todos participan de esta sociedad artificial siempre y cuando tengan las capacidades mínimas para acceder a la misma. Mientras las personas no puedan acceder a un computador, o no conozcan como hacer uso de este, o desconozcan los programas de comunicación en la red, de los lenguajes de comunicación, o cómo acceder e interpretar la información disponible en las redes; mientras no sean empoderadas en estas capacidades, sencillamente no podrán acceder a la información y mucho menos podrán insertar sus actividades a esta sociedad.

En ese sentido la inclusión digital, más que una preocupación mundial relacionada al desenvolvimiento económico, constituye un reto para muchos países. Por ejemplo para los países de América Latina, según el Mapa de la Inclusión Digital (NERI, 2012), es evidente la necesidad de asumir medidas urgentes para mejorar el acceso digital para sus ciudadanos (véase el Gráfico 1).

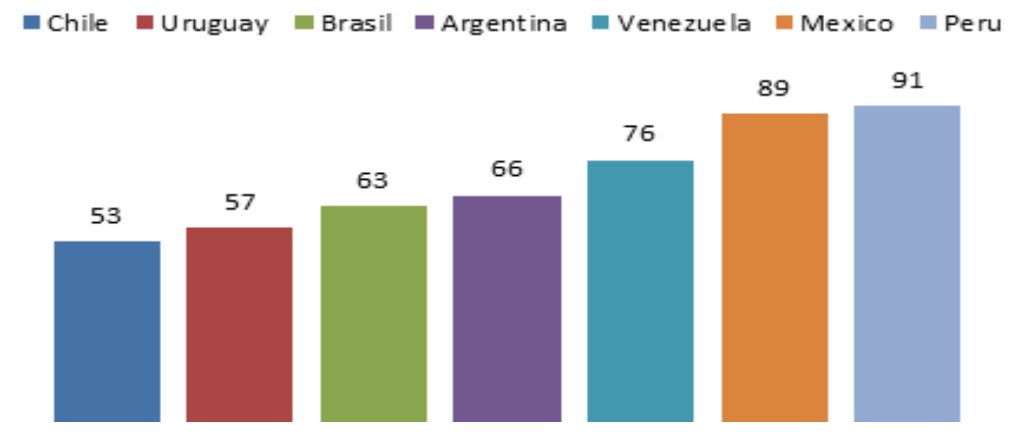

Gráfico 1 - Ranking Mundial de acceso a Internet 2010 Fuente: Elaboración propia 
De acuerdo a Peres y Hilbert (2009, p.304) se pueden reducir los índices de exclusión digital principalmente para incentivar la participación de los países en los mercados internacionales con altos grados de competitividad. Agregan además que:

[...] la brecha digital no solamente se observa entre países sino también dentro de ellos, lo cual refleja y profundiza los problemas de desigualdad distributiva ya existentes en la región y que podrían agravarse aún más si los procesos de transformación que implica la creación de sociedades de la información se basarán únicamente en las fuerzas del mercado. Por ello es necesario contar con políticas públicas de TIC que no solamente apuntan a reducir la brecha digital internacional, sino también a promover una mayor integración social en la que nadie quede excluido de los beneficios de estas tecnologías. (PERES, 2009, p.303)

De otro lado, el énfasis en el uso de las TIC mueve estrategias políticas, desarrollo de productos y servicios, produce el desarrollo de algunas profesiones e configura la práctica de muchas otras que tienen que insertar la tecnología en el cotidiano, como por ejemplo la información y la educación.

\begin{abstract}
[...] Las TIC son herramientas destinadas a optimizar los procesos que sustentan las actividades cotidianas de la sociedad actual; por eso, la creación de las sociedades de la información exige adoptarlas en todos los sectores de la organización económica y social, lo cual, además de implicar la aplicación de políticas transversales de alcance multisectorial, significa que deben promoverse mediante políticas específicas para cada sector donde estén presentes. Puesto que los beneficios de las TIC son el resultado de su capacidad de optimizar los procesos entre diversos agentes intervinientes, no basta con que cada sector las adopte, sino que deben desarrollarse simultáneamente para generar procesos complementarios que faciliten la integración de los procesos productivos y las diversas formas en que se organiza la sociedad (PERES, 2009, p.304)
\end{abstract}

En estos procesos, los desafíos son extensivos a la práctica y actividad de los profesionales de la información, quienes poseen el conocimiento necesario sobre aspectos claves del uso, la organización, la recuperación, la producción y la difusión de la información. Es bajo estas circunstancias que se puede reconocer el rol clave del profesional de la $\mathrm{Cl}$ como mediador, facilitador, intermediario, y agente social de cambio para acceder a la Sociedad del Conocimiento.

\title{
6 La responsabilidad social del profesional de la información
}

Señala Borko (1968, p. 3) que la Cl es una importante disciplina emergente, y el científico de la información tiene un importante función en nuestra sociedad. Y es que la función social de los profesionales de información, como agrega Amorín (2004, p. 5), es asegurar que aquellas personas que necesitan de conocimiento puedan recibirlo, independientemente de haberlo buscado o no.

Así, el profesional de la información posee un rol fundamental en el proceso de mediación entre el usuario y sistemas de información [...] Más que organizar y procesar el conocimiento, será importante proveer su acceso público a través de las más diversas formas y de los más diversos canales de comunicación, de modo que esa nueva fuerza de producción social pueda estar al alcance de sus usuarios potenciales (AMORIN, 2004, p. 5, traducción propia).

Manifiesta Freire (2004a, p. 9), por ejemplo, que los científicos y profesionales de la información podrían desarrollar el papel de mediadores en el proceso de comunicación social, en especial en las situaciones de comunicación del conocimiento de naturaleza técnica y científica para los diversos grupos de la sociedad. Agrega además Paradelo-Luque (2011, p. 98) que el especialista de la información es un profesional que actúa como un gestor del conocimiento, de tal forma que contribuye a la transformación de la información en conocimiento y de este en acción que resulte en riqueza social. Así también manifiesta que la buena práctica de la profesión consiste en la aceptación de estos nuevos principios y valores del ejercicio de la profesión acorde con los cambios experimentados en la sociedad. Sostiene finalmente que llevar a la práctica este conjunto de principios es un reto para el desarrollo de la ética profesional con calidad, que se traducirá luego en acciones que inciden en la buena organización del conocimiento además de la formación de ciudadanos proactivos, participativos y con derechos de acceso de la información.

Es oportuno rescatar para este punto, la Declaración de Buenos Aires esbozada en el 2004 por el Foro Social de Información, Documentación y Bibliotecas, en la cual al referirse a los profesionales de la información, expresa: 
[...] facilitadores del cambio social, formadores de opinión, promotores de la democratización de la información y el conocimiento, gestores educativos y actores comprometidos con los procesos sociales y políticos [...] la teoría como la práctica de la bibliotecología, la documentación y la archivonomía están determinadas por las necesidades que se generan en la estructura social; por ende, la creación y el ejercicio de estas disciplinas y profesiones deben cumplir la misión de fomentar la opinión pública, el juicio crítico, la libre toma de decisiones y contribuir activamente en el combate contra el analfabetismo en todas sus variantes entre la comunidad de sus usuarios con el fin de mejorar la vida y el entorno colectivo o personal de los mismos (FORO SOCIAL DE INFORMACIÓN, DOCUMENTACIÓN Y BIBLIOTECAS, 2004)

De acuerdo a todos estos alcances se sustentan una vez más la importancia de la actividad del profesional de la información en la Sociedad del Conocimiento. Su actuación multidisciplinar en entornos cambiantes, lo acredita para entender y comprender los cambios de rol del conocimiento tanto en los individuos como en las organizaciones y en consecuencia en las culturas.

Complementariamente, el rol de brindar y poner la información a disposición de las personas, debe incluir a todas ellas, en todas sus condiciones, y de manera preferencial a aquellas de menos recursos, o discapacidades. Esto implica también el reto de una capacidad amplia para los profesionales de la información, en el planteamiento de diversos servicios de información que garanticen el acceso para todas las personas, incluyendo aquellas con limitaciones. Para el profesional de la $\mathrm{Cl}$ esto significa considerar que la terminología "inclusión" en una categoría amplia, con tres aspectos a considerar (véase la Figura 1):

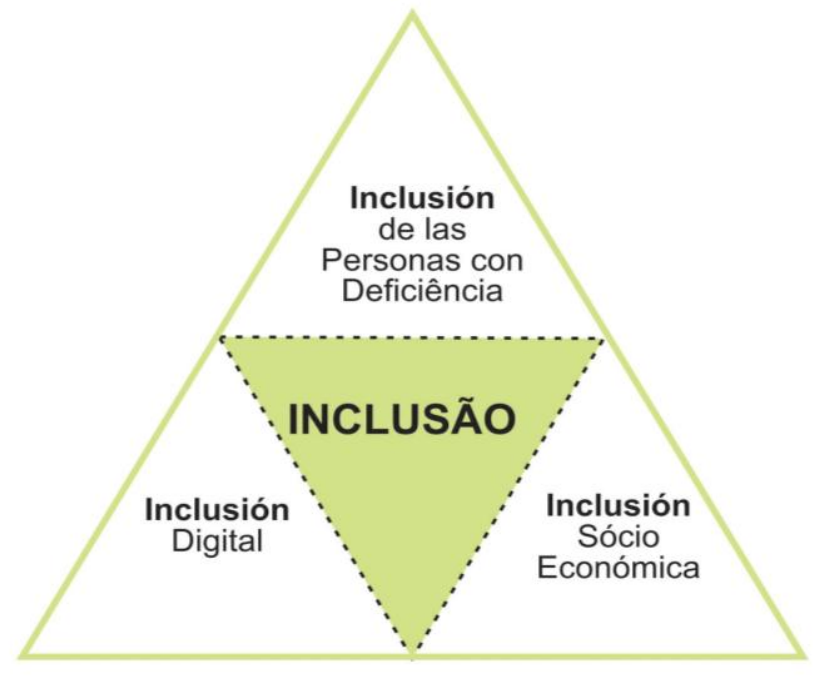

Figura 1 - Pirámide de la Inclusión

Fuente: Elaboración propia

Como un concepto macro, la inclusión se manifiesta en otros aspectos a) uno relacionado a la inclusión social que envuelve políticas públicas de reducción de desigualdades sociales tales como programas de transferencia de rentas, de acceso a bienes y servicios públicos tales como salud, educación; b) una relacionada a inclusión digital que tiene conexión con programas de promoción del uso de las tecnologías, que cuentan con participación directa de profesionales de información en procesos inclusivos utilizando estrategias de mediación, difusión y capacitación c) relacionado a inclusión de personas con deficiencia que envuelve las categorías anteriores y que además presupone programas de accesibilidad física y digital a través de estrategias educativas y tecnologías diferenciadas (tecnologías asistidas).

Al respecto Cuevas-Cerveró e García-Moreno (2010) demuestran la posibilidad de uso de los conceptos Information Literacy, Educación y Salud, que fueron representadas en el Modelo IDEIAS (Inclusión Digital y Educación Informacional para la Salud), como elementos de inclusión social promovidos por el profesional de la información.

El modelo evaluativo que propone Ideias involucra destrezas, conocimientos y actitudes con una clara vocación educativa, y en él se mezclan y entretejen la educación en salud, la 
educación para la información y la formación tecnológica. (CUEVAS-CERVERÓ, GARCIAMORENO, 2010, p.244)

Considerando estas interrelaciones entre las categorías de inclusión, los profesionales de la información pueden actuar, por ejemplo, en el desarrollo de las habilidades informativas para el uso de las tecnologías, parte importante de un proceso de inclusión digital, y como consecuencia de ello, hacer viable la inclusión digital.

En este enfoque y tomando como referencia un escenario común para el profesional de la información, como lo es la biblioteca, Civallero (2012) plantea:

La biblioteca, como gestora de un valioso recurso público (la información), se halla en una encrucijada. Por un lado, al encontrarse dentro de los objetivos de la lucha entre los grupos sociales, debe asegurarse su neutralidad y garantizar que el bien que salvaguarda y distribuye (vital para el desarrollo y el progreso de la sociedad) llegue a todos por igual. Por el otro, debe lograr que la información que maneja sirva como herramienta a aquellos colectivos excluidos que pretendan minimizar las consecuencias de su estado y los bloqueos a los que se ven sometidos. En ambos casos, los profesionales de la bibliotecología y la documentación deberán apelar a su responsabilidad social, su ética profesional y su compromiso para hacer frente a los retos y dificultades que entrañan tan complejas tareas (CIVALLERO, 2012, p.2)

Además sugiere que la formación del profesional de la información se debe orientar para una "bibliotecología progresista" que corresponde a:

[...] una corriente de pensamiento y acción dentro de las ciencias de la información que deja de lado los aspectos técnicos de la profesión para concentrarse en la bibliotecología como fenómeno social, y en el análisis crítico de todos los vínculos entre una unidad de información y la sociedad que la rodea, incluyendo temas como responsabilidad, libertad, equidad y justicia (CIVALLERO, 2012, p.11)

Alves y Vogentim (2013) también plantean la responsabilidad social del profesional de la información a partir de una lógica de promoción e incorporación:

"[...] a sus quehaceres la accesibilidad y la inclusión de personas con deficiencia en sus áreas de actuación, posibilitando asi, a los usuarios en estas condiciones, el contacto, la relación e la apropiación de la información, independiente de la forma como esta ser vinculada: económica, políticas, sociales y culturales (ALVES; VIGENTIM, 2013, p.9)

Tenemos entonces que el ejercicio socialmente responsable de los profesionales de la información se efectiviza a través de las prácticas de mediación de la información que proporciona instrumentos a los ciudadanos en la búsqueda de información independiente de sus condiciones física, intelectuales, sociales o económicas a través de técnicas y procedimientos tales como las que se estructuran en los conceptos de Information Literacy.

Además de eso, es importante considerar que el profesional de la información esta llamado a construir una práctica y una identidad profesional basada en el protagonismo social, proactiva, y en cuyo liderazgo y articulación exprese un sólido compromiso con la inclusión social, donde todas las personas, inclusive aquellas como deficiencias, puedan acceder a la información.

\section{Conclusiones}

Concordamos con los investigadores que fueron recopilados y analizados, en que la Ciencia de la Información tuvo y tiene en la actualidad un rol importante a desempeñar, por su fuerte dimensión social y humana, la cual sobrepasa a la dimensión tecnológica.

Consideramos que la Ciencia de la Información responde directamente a las demandas sociales de la información, las cuales están amparadas en la interdisciplinaridad, en la tecnología, y en la participación en la evolución de la Sociedad de la Información y del Conocimiento. Pero que requiere estar en constante reflexión para no perder su compromiso orientador y transformador en la sociedad y con las personas.

Postulamos que la responsabilidad social de la Ciencia de la Información se refiere al comportamiento ético y las acciones que su comunidad profesional desarrolla en la búsqueda de un equilibrio entre los diversos factores y de nuestra sociedad.

Consideramos que el Acceso a la Información es el eje principal de la responsabilidad social de la Ciencia de la Información y sus profesionales. En ese sentido constituyen parte de sus principales deberes y 
responsabilidades, las acciones en defensa del derecho a la información, el acceso a la información pública, y el desarrollo de capacidades para el manejo de información, entre otras.

Creemos que los profesionales de la Ciencia de la Información son mediadores y facilitadores de la comunicación del conocimiento. Por tanto es necesario que estos científicos y profesionales se involucren en acciones en favor de la ciudadanía, buscando el desarrollo de nuevas formas de inclusión social dirigidas a las poblaciones más vulnerables. Es por ello necesario que la conciencia ética y la sensibilidad social de estos profesionales sea inculcada desde su formación académica proyectando asi su labor hacia la sociedad.

Finalmente, estamos convencidos que la información, en sí misma, es una fuerza de transformación social y que puede ser la vía e instrumento a una sociedad más justa.

\section{Referências}

ALMEIDA, C. C. de; BASTOS,F.M.; BITTENCOURT, F.. Uma Leitura dos Fundamentos Histórico-Sociais da Ciência Da Informação. Revista Eletrônica Informação e Cognição, Marilia, v. 6, n. 1, p. 68-89, 2007. Disponible en $<$ http://www.ofaj.com.br/textos conteudo.php?cod=1>. Acceso en: 01 Dic. 2013.

AMORIM, R. R. A responsabilidade social dos profissionais da informação e a preservação do meio ambiente. In: CONGRESO INTERNACIONAL DE INFORMACIÓN, 2004. Cuba. Anais... Cuba: IDICT, 2004. Disponible en:<http://www.redciencia.cu/empres/Intempres2004/Sitio/Ponencias/3.pdf>. Acceso en: 01 Dic. 2013.

BELKIN, N. J. Information concepts for information science. Journal of Documentation, London, v. 34, n.1, p. 55-85, Mar. 1978.

BORKO, H. Information science: whats is it? American Documentation, v.19, n.1, p.3-5, Jan., 1968.

BUNGE, M.A. Ciencia: Su metodo y su filosofía (la). Buenos aires: Siglo Veinte, 1973. 159 p.

CAPURRO, R.; HJORLAND, B.. O conceito de informação. Perspectivas em Ciência da Informação, Belo Horizonte, v. 12, n. 1, p. 148- 207, jan./abr. 2007.

CASTELLS, M. La era de la información. Economía, sociedad y cultura. Vol. 1: La sociedad red. 2. ed. 4. reimp. Madrid: Alianza Editorial, 2000. 628 p. : Disponible en <http://es.scribd.com/doc/55952938/Castells-La-Sociedad-Red>. Acceso en: 01 Dic. 2013

CIVALLERO, E. El rol de la biblioteca en la inclusión social. In: XIII Jornadas de Gestión de la Información "De la responsabilidad al compromiso social" organizadas por SEDIC (Asociación Española de Documentación e Información) en Madrid, España, los días 17 y 18 de noviembre de 2011. Disponible en:<http://www.sedic.es/Conferencia Edgardo Civallero.pdf> Accesso en 23 Jun. 2015

CUEVAS CERVERÓ, A.; SIMEÃO, E. (Coord.). Alfabetização informacional e inclusão digital: modelo de infoinclusão social. Brasília: Thesaurus, 2011. 219 p. ISBN 9788540900264

CUEVAS CERVERÓ, A.; GARCÍA- MORENO, M. A.. Ideias, un modelo de evaluación para inclusión digital y alfabetización informacional orientado a salud. El profesional de la información, v. 19, n. 3, mayo-junio 2010. Disponible en :<http://www.elprofesionaldelainformacion.com/contenidos/2010/mayo/03.pdf> Acceso en : 23 Jun. 2015.

ENCUENTRO DE CIENCIAS DE LA INFORMACIÓN DEL MERCOSUR ECIM 2015. Disponible en: <http://encuentrocsinfo.com.ar/> Acesso en: 24 Jun. 2015.

FORO SOCIAL DE INFORMACIÓN, DOCUMENTACIÓN Y BIBLIOTECAS. 2004. Declaración de Buenos Aires: sobre información, documentación y bibliotecas. Buenos Aires: El Foro, 2004. 1 p. Disponible en <http://www.ofaj.com.br/textos conteudo.php?cod=1>. Acceso en: 01 Dic. 2013.

FREIRE, I.M. A responsabilidade social da Ciência da Informação na perspectiva da consciência possível. DataGramaZero, Río de Janeiro, v. 5, n. 1, p. 00, fev. 2004. Disponible en <http://www.dgz.org.br/fev04/Art 02.htm>. Acceso en: 02 May. 2013.

FREIRE, I.M. O desafio da inclusão digital. Transinformação, Campinas, v. 16, n. 2, p. 189-194, maio/ago. 2004. Disponible en <http://periodicos.puc-campinas.edu.br/seer/index.php/transinfo/article/view/720/700>. Acceso en: 02 Jul. 2013.

FREIRE, P. Educación y Cambio.Cuco: Buenos Aires, 2002. Disponible en:<http://simeduco.org/wpcontent/uploads/2015/04/Educacion-y-Cambio.pdf> Acceso en: 23 jun. 2015.

INTERNATIONAL STANDARD ORGANIZATION. ISO 26000:2010: Guidance on social responsibility, 2010.106 p. 
INTERNATIONAL FEDERATION OF LIBRARY ASSOCIATIONS AND INSTITUTIONS. Declaración de la IFLA sobre las bibliotecas y la libertad intelectual. La Haya: IFLA, 1999.

LE COADIC, Y.F. A Ciência da Informação. Brasília: Briquet de Lemos, 1996.

NERI, Marcelo Côrtes (coord.). Mapa da inclusão digital. Rio de Janeiro: Fundação Getúlio Vargas, Centro de Políticas Sociais, 2012. Disponible en:< http://www.cps.fgv.br/cps/bd/mid2012/MID FT FGV CPS Neri TextoPrincipal Fim GRAFICA fim.pdf $>$ Acceso en: 23 jun. 2015.

MÚNERA TORRES, M.T. Responsabilidad social del profesional de la Ciencia de la Información. Revista EDICIC, Marília, v. 1, n. 1, p. 135-147. 2011. Disponible en

$<$ http://www.edicic.org/revista/index.php?journal=RevistaEDICIC\&page=article\&op=download\&path\%5B\%5D=14\&path\%5B \%5 $\mathrm{D=pdf}>$. Acceso en: 05 Dic. 2013.

NIETZSCHE, F. La genealogía de la moral, Madrid, Alianza Editorial, [1887] 1996.

ORGANIZACIÓN DE AS NACIONES UNIDAS. Objetivos de Desarrollo del Milenio. Washington: Programa de las Naciones Unidas para el Desarrollo, 2000. Disponible en:< http://www.un.org/es/millenniumgoals/> Acceso en: 23 jun. 2015.

ORGANIZAÇÃO DAS NAÇÕES UNIDAS. Declaração Universal dos Direitos Humanos da Organização das Nações Unidas. Washington: ONU, 1948. Disponible en:<http://www.dudh.org.br/wp-content/uploads/2014/12/dudh.pdf> Acceso en: 23 jun. 2015.

PARADELO-LUQUE, A. ; CUVERTINO, P. Conducta ética, actitudes y compromiso del profesional de la información en la sociedad del conocimiento. Revista EDICIC, Marília, v. 1, n. 1, p. 92-102. 2011. Disponible en $<$ http://www.edicic.org/revista/index.php?journal=RevistaEDICIC\&page=article\&op=view\&path\%5B $\% 5 \mathrm{D}=11 \&$ path $\% 5 \mathrm{~B} \% 5 \mathrm{D}=\mathrm{pd}$ f>. Acceso en: 05 Dic. 2013.

PERES, W; HILBERT, M.R. La sociedad de la información en América Latina y el Caribe : desarrollo de las tecnologías y tecnologías para el desarrollo. Santiago de Chile : Naciones Unidas, CEPAL, 2009., 2009. (Libros de la CEPAL: 98). ISBN: 9789213231777.

RENAULT, L.V.; MARTINS, R. O retrato da ciência da informação: uma análise de seus fundamentos sociais. Encontros Bibli: Revista Eletrônica de Biblioteconomia e Ciência da Informação, Florianópolis, v. 12, n. 23, p. 133-150, 1ํ sem. 2007. Disponible en <http://www.brapci.ufpr.br/download.php?dd0=11995>. Acceso en: 05 Dic. 2013.

SARACEVIC, T. Ciência da informação: origem, evolução e relações.Perspectivas em Ciência da Informação, Belo Horizonte, v. 1, n. 1, p. 41-62, jan./jun. 1996. Disponible en <http://www.brapci.ufpr.br/download.php?dd0=11621>. Acceso en: 04 Dic. 2013.

TOMANIK, E. A. O olhar no espelho: "conversas" sobre a pesquisa em ciências sociais. 2. ed. rev. Maringá: Eduem, 2004.

THOMPSON, J. B. A Mídia e a modernidade: uma teoria social da mídia. Tradução de Wagner Oliveira. Petrópolis: Vozes, 1998.

UNESCO. Hacia las sociedades del conocimiento: Informe mundial de la UNESCO. París: Ediciones UNESCO, 2005. Disponible en: <http://unesdoc.unesco.org/images/0012/001255/125506s.pdf\#page=55>. Acceso en: 05 Dic. 2013.

WERSIG, G.; NEVELING, U. The phenomena of interest to information science. Information Scientist, v.9, n.4, p. 127-140, Dec. 1975. Versão traduzida para o português por Tarcísio Zandonade: Wersig \& Neveling. Os fenômenos de interesse para a ciência da Informação.

\section{Datos de las autoras}

\section{Ada Suyin Sosa Solano}

Magister en Gerencia Social - Pontificia Universidad Católica del Perú - PUCP (2010). Especialista en Gestión de Proyectos Sociales - Pontificia Universidad Católica del Perú - PUCP (2007). Graduada en Bibliotecología y Ciencias de la Información - Universidad Nacional Mayor de San Marcos - UNMSM (2002). Se ha desenpeñado como bibliotecóloga en el Sistema de Bibliotecas de la Pontificia Universidad Católica del Perú. Ha sido 
facilitadora de talleres en la Asociación Jatun Nani. Sus áreas de interés son: lectura y biblioteca, ciudadanía, mujeres, y políticas públicas. Actualmente es estudiante en el Programa de Pós-graduação em Ciência da Informação de la Universidade de Brasília - UnB, y miembro del Grupo de Pesquisa Comunicação Científica e Mediação de esta universidad.

ada.sosa@gmail.com

\section{Celia Revilanda Costa}

Magister en Educación por la Universidad Federal de Piauí - UFPI(2008). Graduada en Pedagogía por la Universidade Federal de Goiás - UFG (1994). Tiene experiencia en el área de Educación en Derechos Humanos, actuando principalmente en los siguientes temas: diversidad, escuela, comunidad, mass media y juventud. Actualmente es estudiante en el Programa de Pós-graduação em Ciência da Informação de la Universidade de Brasília - UnB, y miembro del Grupo de Pesquisa Comunicação Científica e Mediação de esta universidad.

\section{celiarevilandia@gmail.com}

\section{Elmira Luzia Melo Soares Simeão}

Doctora en Ciência da Informação por la Universidade de Brasília - UnB (2003). Magister en Comunicação e Cultura por la Universidade Federal do Rio de Janeiro - UFRJ (1998). Graduada en Comunicação Social pela Universidade Federal do Piauí - UFPI (1990). Actúa en el área de publicación, formación de acervos y calificación informacional. Desde el 2010 ejerce la dirección de la Faculdade de Ciência da Informação da Universidade de Brasília, siendo miembro del Conselho de Ensino e Pesquisa da UnB (CEPE), Conselho de Administração (CAD) e Conselho Superior da UnB (CONSUNI). Es profesora en la Faculdade de Ciência da Informação - FCl, en la graduación de Biblioteconomia y en el Programa de Pós-Graduação em Ciência da Informação da Universidade de Brasília.

elmirasimeao@gmail.com

$\begin{array}{ll}\text { Recibido - Received } & : 2015-03-26 \\ \text { Aceptado-Accepted } & : 2015-06-26\end{array}$

\section{$(\mathrm{cc}) \mathrm{EY}$}

This work is licensed under a Creative Commons Attribution 4.0

United States License.

\section{UILIS D-Solfel}

This journal is published by the University Library System of the University of Pittsburgh as part of its D-Scribe Digital Publishing Program and is cosponsored by the University of Pittsburgh Press. 